\title{
Psicooncología
}

ISSN: 1696-7240

http://dx.doi.org/10.5209/PSIC.59171

\section{Estoicismo frente al cáncer: riesgo o protección}

\author{
Beatriz Castelo, ${ }^{1, *}$; Ángela Palao²; Alberto Carmona-Bayonas ${ }^{3}$; Paula Jiménez-Fonseca \\ Carlos Jara ${ }^{5}$; Francisco Ayala ${ }^{6}$; Ismael Ghanem; Oliver Higuera ${ }^{7}$; Raquel Hernández ${ }^{9}$; \\ Caterina Calderón ${ }^{10}$
}

Recibido: 4 de diciembre de 2017 / Aceptado: 23 de febrero de 2018

Resumen. Detectar una actitud de afrontamiento estoica en los pacientes con cáncer es importante porque puede favorecer o dificultar el tratamiento oncológico. Objetivo: En este trabajo, analizamos si en función de la edad, el género, la presencia de dolor y el tiempo transcurrido desde que empezaron los síntomas hasta el diagnóstico del cáncer, podemos asignar a las conductas estoicas un valor positivo o negativo, como factor de protección o de riesgo en los pacientes. Material y Métodos: evaluamos 540 pacientes que acudieron a la consulta de Oncología Médica para valoración de tratamiento adyuvante tras una cirugía oncológica, entre junio del 2015 y diciembre del 2016.

1 Beatriz Castelo. Servicio de Oncología Médica.

Hospital Universitario La Paz. Madrid. Po de la Castellana, 26128046.

E-mail: castelobeatriz@gmail.com

2 Ángela Palao. Servicio de Psiquiatria.

Hospital Universitario La Paz. Madrid. Po de la Castellana, 26128046.

E-mail: apalaotarrero@gmail.com

3 Alberto Carmona-Bayonas. Servicio de Hematología y Oncología Médica.

Hospital Universitario Morales Meseguer de Murcia. Murcia.

E-mail: alberto.carmonabayonas@gmail.com

4 Paula Jiménez-Fonseca. Servicio de Oncología Médica. Hospital Universitario Central de Asturias. Oviedo.

E-mail: palucaji@hotmail.com

5 Carlos Jara Sánchez Hospital Universitario Fundación Alcorcón - Universidad Rey Juan Carlos. Madrid.

E-mail: cjara@fhalcorcon.es

6 Francisco Ayala. Servicio de Hematología y Oncología Médica.

Hospital Universitario Morales Meseguer de Murcia. Murcia

E-mail: frayala@um.es

7 Ismael Ghanem Servicio de Oncología Médica. Hospital Universitario La Paz.

Madrid. $\mathrm{P}^{\circ}$ de la Castellana, 26128046.

E-mail: isma g c@hotmail.com

8 Oliver Higuera Servicio de Oncología Médica. Hospital Universitario La Paz.

Madrid. P $^{\mathrm{o}}$ de la Castellana, 26128046.

E-mail: oliverhiguera@gmail.com

9 Raquel Hernández. Servicio de Oncología Médica. Hospital Universitario de Canarias. Tenerife.

E-mail: raquel_hsg@hotmail.com

10 Caterina Calderón. Servicio de Psicología Clínica y Psicobiología. Facultad de Psicología.

Universidad de Barcelona. Barcelona.

E-mail: ccalderon@ub.edu

* Dirección de correspondencia: Beatriz Castelo. Servicio de Oncología Médica.

Hospital Universitario La Paz. Madrid. Po de la Castellana, 26128046.

E-mail: castelobeatriz@gmail.com 
Los datos clínicos y demográficos se obtuvieron a través de la entrevista médica y fueron: género, edad, estado civil, nivel educativo, área ocupacional, localización del tumor, estadio y tiempo trascurrido desde la presencia de los síntomas y la consulta al médico. Se administraron en papel y presencialmente los siguientes cuestionarios: Inventario de Dolor Breve, Brief Symptom Inventory y Liverpool Stoicism Scale. Resultados: Los hombres de la muestra mostraron casi 5 veces más probabilidad de presentar altas puntuaciones en estoicismo que las mujeres y 7 veces más si se asocia un estado de ánimo decaído y edad avanzada. El estoicismo fue mayor en los pacientes de más edad. No se ha hallado relación entre el estoicismo y las escalas de dolor. Conclusión: Las tres características que discriminan mejor entre los pacientes con altas y/o bajas puntuaciones en estoicismo son el género, la edad y el estado de ánimo depresivo. El hecho de que el estoicismo esté asociado a hombres ancianos con depresión nos hace pensar que el estoicismo debe ser considerado más como un factor de riesgo que de protección. La madurez relacionada con la edad conlleva inevitablemente una mayor aceptación de las pérdidas y una propensión a ocultar el dolor, o a no buscar ayuda para evitar mostrar debilidad. Ante esta situación es importante contar con la información que nos proporciona la familia y reconocer la importancia del apoyo familiar sobre a estas personas que pueden ser de entrada más vulnerables.

Palabras clave: afrontamiento; depresión; dolor; estoicismo; riesgo.

\title{
[en] Stoicism against cancer: risk or protection
}

\begin{abstract}
Detecting an attitude of stoic coping in patients with cancer is important because it can favor or hinder oncological treatment. Objective: In this work, we analyze whether depending on age, gender, the presence of pain and the time elapsed since the symptoms began to the diagnosis of cancer, we can assign a positive or negative value to the stoic behaviors as a factor of protection or risk in patients. Material and Methods: We evaluated 540 patients who attended the Medical Oncology consultation for evaluation of adjuvant treatment after oncological surgery, between June 2015 and December 2016. The clinical and demographic data were obtained through the medical interview and were: gender, age, marital status, educational level, occupational area, location of the tumor, stage and time elapsed since the presence of symptoms and consultation with the doctor. The following questionnaires were administered on paper and in person: Brief Pain Inventory, Brief Symptom Inventory and Liverpool Stoicism Scale. Results: The men in our sample are almost 5 times more likely to have high stoicism scores than women and 7 times more likely to be associated with a declining state of mind and advanced age. Stoicism was greater in older patients. No relationship has been found between stoicism and pain scales. Conclusion: The three characteristics that best discriminate between patients with high and / or low scores in stoicism are gender, age and depressed mood. The fact that stoicism is associated with elderly men with depression makes us think that the Stoicism should be considered more as a risk factor than as a protection factor. Age-related maturity inevitably leads to greater acceptance of losses and a propensity to hide pain, or not seeking help to avoid showing weakness. Given this situation, it is important to have the information provided by the family and recognize the importance of family support for these people who may be more vulnerable at the outset.
\end{abstract}

Keywords: coping, depression, pain, stoicism, risk.

Sumario. 1. Introducción 2. Método 2.1. Participantes 2.2. Instrumentos de evaluación 2.3. Procedimiento 3. Análisis estadístico 4. Resultados 4.1. Características de los pacientes 4.2. Diferencias en estoicismo y síntomas psicológicos según edad y género 4.3. Relación entre estoicismo y variables en estudio 5. Discusión 6. Conclusiones 7. Referencias bibliográficas.

Cómo citar: Castelo B, Palao Á, Carmona-Bayonas A, Jiménez-Fonseca P, Jara C, Ayala F, Ghanem I, Higuera $\mathrm{O}$, Hernández R, Calderón C. Estoicismo frente al cáncer: riesgo o protección. Psicooncología 2018;15:11-22. Doi: 10.5209/PSIC.59171. 
"Ni siquiera la muerte tiene gran importancia por sí misma.

Es nuestro concepto de la muerte, nuestra idea, lo que es terrible, lo que nos aterroriza" (Epicteto, Enchiridon)

\section{Introducción}

El cáncer es una de las enfermedades más importantes de nuestra época, tanto por su elevada incidencia como por las consecuencias del mismo, constituyendo uno de los principales retos sanitarios. Ante esta grave enfermedad que genera mucha incertidumbre, no todos los pacientes reaccionan del mismo modo, ni generan el mismo tipo de demanda a los profesionales de la salud. Mientras unos expresan con gran emoción el miedo que sienten ante el reto al que se enfrentan, otros muestran serenidad y conformidad. A esta última actitud se la denomina estoicismo ${ }^{(1)}$, siguiendo la senda de un grupo de filósofos de la antigüedad que plantearon responder de forma racional y controlada tanto ante las adversidades como ante las fortunas de la vida, haciendo hincapié en diferenciar lo que estaba sujeto a nuestro control de lo que no lo estaba ${ }^{(2)}$ minimizando las respuestas emocionales y de este modo fomentando la virtud.

En este sentido, se nos presenta el estoicismo como una de las tres escuelas filosóficas postaristotélicas, que junto con el epicureísmo y el escepticismo sostenían que el fin del ser humano es la felicidad, y que la felicidad consiste en la ausencia de turbación y en la eliminación de alteraciones. Epicteto, filósofo estoico, nos invita a poner la atención en nuestros actos, pero también en aquello que provocan nuestras acciones, es decir, nuestra carga subjetiva de creencias, pensamientos, prejuicios, ideas o deseos. Los acontecimientos no nos hacen daño, pero nuestra visión de los mismos nos lo puede hacer. Son nuestras actitudes y reacciones las que nos causan problemas. Los filósofos estoicos invitan, pues, a mirar hacia dentro, a tomar conciencia de nuestros pensamientos y evaluarlos críticamente con el auxilio de una razón crítica.

En general las conductas estoicas se atribuyen al género masculino y a las personas mayores, pues suelen ser los colectivos que expresan en menor medida sus emociones ${ }^{(3-5)}$. Los hombres temen parecer poco viriles si se quejan o bien paradójicamente, temen encontrarse ante una enfermedad grave y por eso retrasan la visita médica ${ }^{(6)}$. Asimismo, hay autores que consideran que con la edad se madura y se tiende a ser más estoico ${ }^{(7)}$ aceptando mejor las pérdidas; otros lo atribuyen a las condiciones contextuales adversas que vivieron los actuales ancianos en su infancia y juventud (guerra civil, postguerra), experiencias que les fortalecieron ante la adversidad y que crean cierto efecto de cohorte ${ }^{(8,9)}$. Podemos pues describir el estoicismo como un estilo de respuesta que guarda relación con la personalidad del individuo, pero también con determinadas características contextuales, en nuestro caso, como un estilo emocional a modo de estrategia de afrontamiento frente al cáncer.

Como médicos y profesionales de la salud, deseamos conocer si las respuestas estoicas son verdaderamente beneficiosas para el paciente, tal como plantea esta escuela clásica, los neoestoicos renacentistas, o autores de la talla de Ellis o Beck. Nuestro modelo comunicativo y empático en las consultas debe reflejar nuestro posicionamiento respecto a este fenómeno tan presente en la práctica clínica diaria. 
Por este motivo, nos planteamos analizar las diferencias entre pacientes con alto o bajo estoicismo en función de la edad, género, presencia de dolor y el tiempo transcurrido desde que empezaron los síntomas hasta el diagnóstico del cáncer, con el objetivo de establecer si podemos asignar a las conductas estoicas un valor positivo o negativo, como factor de protección o de riesgo en los pacientes.

\section{Método}

\subsection{Participantes}

Los criterios de inclusión fueron: tener 18 o más años, diagnóstico de un cáncer sólido histológicamente confirmado, sometido a cirugía con intención curativa y continuar en el centro donde se realiza la primera visita durante todo el tratamiento adyuvante. Y como criterios de exclusión: presentar enfermedad metastásica, haber sido tratado con radioterapia o quimioterapia preoperatoria, resección tumoral incompleta, gozar de condiciones físicas, comorbilidades y/o edad que constituyan una contraindicación absoluta a la quimioterapia, o padecer alguna enfermedad psiquiátrica grave.

Se seleccionó población oncológica libre de enfermedad, cáncer, debido a que los pacientes habían sido sometidos a cirugía a lo largo del mes anterior, y para quienes el enfoque de la consulta oncológica era decidir sobre la conveniencia de la quimioterapia adyuvante.

\subsection{Instrumentos de evaluación}

Los datos clínicos y demográficos se obtuvieron a través de la entrevista médica y fueron: género, edad, estado civil, nivel educativo, área ocupacional, localización del tumor, estadio y tiempo trascurrido desde la presencia de los síntomas y la consulta al médico.

Se administraron en papel y presencialmente los siguientes cuestionarios:

Inventario de Dolor Breve (Cleeland \& Ryan, 1994), (BPI, siglas en inglés) ${ }^{(10)}$ es un cuestionario auto-administrado, breve y de fácil comprensión que evalúa dos dimensiones: la intensidad del dolor y su impacto en las actividades de la vida del paciente. El BPI es ampliamente utilizado tanto en investigación como en la práctica clínica con pacientes oncológicos ${ }^{(11)}$. Presenta una buena fiabilidad test-retest en muestra española ${ }^{(12)}$.

Brief Symptom Inventory (Derogatis, 2001) (BSI-18, siglas en inglés) ${ }^{(13)}$ es un cuestionario breve de 18 ítems que evalúa tres dimensiones (somatización, depresión y ansiedad), ofreciendo además una puntuación global sobre el Malestar Psicológico (GSI). La dimensión somatización (seis ítems) evalúa el malestar producido por la percepción de problemas físicos. La dimensión de depresión (seis ítems) describe los síntomas relacionados con la depresión, apatía, y tristeza. La dimensión de ansiedad (seis ítems) analiza los sentimientos de miedo, nerviosismo o pánico. Cada ítem se evalúa en una escala tipo Likert de cinco puntos que va desde "nunca" (0) a "extremadamente" (4). La fiabilidad test-retest varía de 0,78 a 0,90 (14).

Liverpool Stoicism Scale (Wagstaff \& Rowledge, 1995) (LSS, siglas en inglés) ${ }^{(1)}$ es un cuestionario que evalúa el estoicismo y que consta de 20 ítems que analizan la 
falta de compromiso emocional, la aversión por expresar abiertamente las emociones y la capacidad de soportarlas. Las respuestas se registraron en una escala Likert de 5 puntos que va de "muy en desacuerdo" (1) a "muy de acuerdo" (5). Las puntuaciones más altas indican mayores niveles de estoicismo y permiten distinguir entre pacientes con alto o bajo estoicismo. La versión original tiene una buena consistencia interna, alfa de Cronbach $=0,83^{(1)}$.

\subsection{Procedimiento}

Los pacientes que acudieron a consulta al oncólogo tras la resección del tumor, entre junio del 2015 y diciembre del 2016, fueron informados del objetivo y procedimiento del estudio. Aquellos que decidieron participar voluntariamente en el estudio, firmaron el consentimiento informado, garantizando el anonimato y confidencialidad de la información de acuerdo con las normas éticas de investigación de la Declaración de Helsinki ${ }^{(15)}$ y la Ley Orgánica 15/1999, de 13 de diciembre, de Protección de Datos de Carácter Personal. A los pacientes se les entregaban los cuestionarios psicosociales que contestaban en ese momento y/o retornaban en la siguiente visita. El proyecto fue revisado en sus aspectos éticos y técnicos por los Comités Éticos de Investigación Clínica (CEIC) de cada una de las instituciones que participaron.

\section{Análisis estadístico}

Inicialmente se realizó un análisis descriptivo de la muestra, con la presentación de frecuencias, medias, medianas y desviaciones típicas de las variables sociodemográficas y clínicas en estudio. Para los análisis estadísticos se tuvo en cuenta el cumplimiento de los supuestos paramétricos. Para explorar la relación entre edad, estoicismo, síntomas psicológicos y dolor en función del género, se calcularon los coeficientes de correlación de Pearson. Para los contrastes de comparación de medias entre grupos en las variables analizadas se utilizó la Prueba $\mathrm{T}$ de Student. Finalmente, se realizó un análisis de regresión logística para determinar la probabilidad de que un paciente presentase altas puntuaciones en estoicismo, sobre la base de las variables en estudio (edad, género, síntomas psicológicos, dolor y tiempo desde el inicio de los síntomas y la visita al médico). Para los análisis se utilizó el paquete estadístico SPSS IBM v23.0 (SPSS Inc., Chicago, IL) para Windows.

\section{Resultados}

\subsection{Características de los pacientes}

Se incluyeron 540 pacientes (61,7\% mujeres) de 13 hospitales españoles. La edad media fue de 58,6 años (desviación típica $(D T)=12,2$ ). En nuestra muestra, las mujeres tenían, de promedio, 7 años menos que los hombres $\left(\mathrm{t}_{(538)}=6,801, \mathrm{p}<0,001\right)$. El $37,8 \%$ no tenía estudios, el $22 \%$ tenía estudios primarios, el $24 \%$ había cursado 
la enseñanza secundaria o formación profesional, el 16\% estudios universitarios y el $57,8 \%$ no trabajaba. El 41,9\% correspondía a pacientes con cáncer de colón, $34,3 \%$ a cáncer de mama, 6,9\% a cáncer de estómago y el 17\% se distribuían en otros tumores. El 16,3\% de los cánceres se encontraba en estadio I, el 36,2\% en estadio II y el $47,6 \%$ en estadio III. Todos recibieron tratamiento complementario a la cirugía con quimioterapia, y el 35,9\% recibió además radioterapia adyuvante. El tiempo promedio entre el inicio de los síntomas y el diagnóstico del cáncer fue de 106 días. Los hombres, de promedio, solían tardar 52 días más que las mujeres en ir al médico cuando notaban los primeros síntomas de la enfermedad $\left(\mathrm{t}_{(433)}=2,178, \mathrm{p}=\right.$ 0,030). Las características sociodemográficas y clínicas de los pacientes se muestran en la Tabla 1.

Tabla 1. Características sociodemográficas y clínicas de los pacientes $(\mathrm{n}=540)$

\begin{tabular}{|c|c|c|}
\hline Características & $n$ & $\%$ \\
\hline Edad (años, Media; DT) & 58,6 & 12,2 \\
\hline \multicolumn{3}{|l|}{ Género } \\
\hline Mujer & 333 & 61,7 \\
\hline Hombre & 207 & 38,3 \\
\hline \multicolumn{3}{|l|}{ Educación } \\
\hline Sin estudios & 204 & 37,8 \\
\hline Estudios primarios & 119 & 22,0 \\
\hline Estudios secundarios & 130 & 24,1 \\
\hline Estudios universitarios & 87 & 16,1 \\
\hline \multicolumn{3}{|l|}{ Ocupación } \\
\hline Activo & 228 & 42,2 \\
\hline Jubilado o desempleado & 312 & 57,8 \\
\hline \multicolumn{3}{|l|}{ Estado civil } \\
\hline Casado/en pareja & 415 & 76,9 \\
\hline Separado/soltero o viudo & 125 & 23,1 \\
\hline \multicolumn{3}{|l|}{ Localización tumor primario } \\
\hline Colon & 226 & 41,9 \\
\hline Mama & 185 & 34,3 \\
\hline Estómago & 37 & 6,9 \\
\hline Otros & 92 & 17,0 \\
\hline \multicolumn{3}{|l|}{ Estadio } \\
\hline I & 81 & 16,3 \\
\hline II & 195 & 36,2 \\
\hline III & 257 & 47,6 \\
\hline Radioterapia adyuvante & 194 & 35,9 \\
\hline Tiempo hasta consulta (días, Media; DT) & 106 & 249 \\
\hline
\end{tabular}

Abreviaturas: N: número; DT: Desviación Típica; \%: Porcentaje 


\subsection{Diferencias en estoicismo y síntomas psicológicos según edad y género}

En la tabla 2 se recoge la relación entre la edad en años y el estoicismo, los síntomas psicológicos, el dolor y el tiempo transcurrido desde la presencia de síntomas y la consulta al médico, considerando además el género. Los resultados muestran principalmente una relación entre la edad y los síntomas psicológicos. Los hombres, a mayor edad, presentan menor impacto psicológico tras el diagnóstico de cáncer, con menos malestar psicológico $(\mathrm{r}=-0,230, \mathrm{p}<0,001)$, ansiedad $(\mathrm{r}=$ $-0,182, \mathrm{p}<0,001)$ y depresión $(\mathrm{r}=-0,296, \mathrm{p}<0,001)$. Las mujeres, con la edad, también manifiestan menor malestar psicológico $(r=-0,119, \mathrm{p}<0,001)$, ansiedad $(\mathrm{r}=-0,173, \mathrm{p}<0,001)$ y somatización $(\mathrm{r}=-0,132, \mathrm{p}<0,001)$, y también son más estoicas $(\mathrm{r}=0,364, \mathrm{p}<0,001)$.

Tabla 2. Correlación entre estoicismo, síntomas psicológicos y dolor según el género

\begin{tabular}{lcc}
\hline Características & $\begin{array}{c}\text { Varones } \\
\mathbf{n = 2 0 7}\end{array}$ & $\begin{array}{c}\text { Mujeres } \\
\mathbf{n}=\mathbf{3 3 3}\end{array}$ \\
\hline LSS- Estoicismo & $-0,038$ & $0,364 * *$ \\
BSI- Malestar psicológico & $-0,230 * *$ & $-0,119^{*}$ \\
BSI- Somatización & $-0,116$ & $-0,132 *$ \\
BSI- Ansiedad & $-0,182^{* *}$ & $-0,173^{* *}$ \\
BSI- Depresión & $-0,296 * *$ & $-0,018$ \\
BPI- Dolor & 0,120 & 0,007 \\
Tiempo hasta la consulta & $-0,104$ & $-0,070$ \\
\hline Abreviaciones: N: número. Nota. ${ }^{*} \mathrm{p}<0,05 ; * * \mathrm{p}<0,001$ &
\end{tabular}

\subsection{Relación entre estoicismo y variables en estudio}

El análisis de las variables clínicas en relación con el estoicismo muestra que las personas que puntúan alto en estoicismo son las de mayor edad $\left(\mathrm{t}_{(307)}=-6,495\right.$, $\mathrm{p}<0,001)$, las que presentan un estado de ánimo más decaído $\left(\mathrm{t}_{(307)}=-2,132\right.$, $\mathrm{p}=0,034)$ y las que suelen tardar más tiempo entre la aparición de los síntomas y la visita al médico $\left(\mathrm{t}_{(246)}=-2,710, \mathrm{p}=0,007\right)$. No se halló relación entre el estoicismo y el dolor (ver tabla 3).

El análisis de regresión logística nos indica que el estoicismo alto vendría explicado por el género (hombres), la edad avanzada y la presencia de síntomas de depresión (Wald $=40,721, \mathrm{p}<0,001$ ). Los hombres tienen casi 5 veces más probabilidad de presentar altas puntuaciones en estoicismo que las mujeres. Si, además, estos hombres son mayores (Wald $=22,836, \mathrm{p}<0,001$ ) y presentan un estado de ánimo decaído (Wald $=11,692, \mathrm{p}<0,001$ ), la probabilidad de presentar altas puntuaciones en estoicismo es 7 veces más que en las mujeres. Estas son las tres características que discriminan mejor entre los pacientes con altas y/o bajas puntuaciones en estoicismo, género, edad y estado de ánimo depresivo (ver tabla 4). 
Tabla 3. Diferencias de medias según estoicismo (bajo y alto), edad, síntomas psicológicos y dolor

\begin{tabular}{lllc}
\hline Variables & \multicolumn{1}{c}{$\begin{array}{c}\text { Estoicismo } \\
\mathbf{b a j o}(\mathbf{p c}<\mathbf{2 5}) \\
\mathbf{n = 2 3 1}\end{array}$} & $\begin{array}{c}\text { Estoicismo } \\
\mathbf{a l t o}(\mathbf{p c}>\mathbf{7 5}) \\
\mathbf{n = 1 7 0}\end{array}$ & $\mathbf{t}$ \\
\hline Edad & $52,9(12,4)$ & $61,7 / 11,3)$ & $-6.495^{* *}$ \\
BSI- Malestar psicológico & $65,2(6,4)$ & $65,0(7,0)$ & 0,364 \\
BSI- Somatización & $62,4(6,9)$ & $61,6(6,8)$ & 0,963 \\
BSI- Ansiedad & $63,3(7,7)$ & $62,2(7,7)$ & 1,344 \\
BSI- Depresión & $60,7(5,5)$ & $62,1(6,3)$ & $-2,132^{*}$ \\
BPI- Dolor & $2,9(0,2)$ & $2,9(0,2)$ & 0,852 \\
Tiempo hasta la consulta & $62,8(78,5)$ & $105,2(13,6)$ & $-2,710^{* *}$ \\
\hline
\end{tabular}

Abreviaturas: N: número; pc: percentil. Nota. ${ }^{*} \mathrm{p}<0,05 ; * * \mathrm{p}<0,001$

Tabla 4. Análisis de regresión logística para la predicción de puntuaciones altas y/o bajas en estoicismo

\begin{tabular}{|c|c|c|c|c|c|c|c|c|}
\hline & & \multirow{2}{*}{ B } & \multirow{2}{*}{$\begin{array}{c}\text { Error } \\
\text { estándar }\end{array}$} & \multirow{2}{*}{ Wald } & \multirow{2}{*}{ Sig. } & \multirow{2}{*}{$\operatorname{Exp}(B)$} & \multicolumn{2}{|c|}{ IC 95\% EXP(B) } \\
\hline & & & & & & & Inferior & Superior \\
\hline \multirow[t]{2}{*}{ Paso 1} & Hombre & 1,763 & 0,276 & 40,721 & 0,000 & 5,829 & 3,392 & 10,018 \\
\hline & Constante & $-0,410$ & 0,147 & 7,771 & 0,005 & 0,664 & & \\
\hline \multirow[t]{3}{*}{ Paso 2} & Hombre & 1,538 & 0,288 & 28,539 & 0,000 & 4,656 & 2,648 & 8,186 \\
\hline & Edad & 0,050 & 0,011 & 21,297 & 0,000 & 1,051 & 1,029 & 1,074 \\
\hline & Constante & $-3,201$ & 0,631 & 25,753 & 0,000 & 0,041 & & \\
\hline \multirow[t]{4}{*}{ Paso 3} & Hombre & 1,700 & 0,299 & 32,399 & 0,000 & 5,474 & 3,048 & 9,830 \\
\hline & Edad & 0,054 & 0,011 & 22,836 & 0,000 & 1,055 & 1,032 & 1,078 \\
\hline & Depresión & 0,079 & 0,023 & 11,692 & 0,001 & 1,082 & 1,034 & 1,132 \\
\hline & Constante & $-8,319$ & 1,662 & 25,060 & 0,000 & 0,000 & & \\
\hline
\end{tabular}

Abreviaturas: IC: intervalo de confianza, sig: significación.

\section{Discusión}

El objetivo principal de nuestro estudio ha sido analizar las diferencias entre pacientes con alto o bajo estoicismo en función de la presencia de dolor, síntomas psicológicos, edad y tiempo transcurrido desde que empezaron los síntomas hasta el diagnóstico del cáncer. El género, la edad y la depresión fueron las tres variables que mejor discriminaron entre pacientes con altas y/o bajas puntuaciones en estoicismo. Los hombres de nuestra muestra presentan casi 5 veces más probabilidad de presentar altas puntuaciones en estoicismo que las mujeres y 7 veces más si se asocia un estado de ánimo decaído y edad avanzada. El hecho de que el estoicismo esté asociado a hombres ancianos con depresión nos hace pensar que el estoicismo debe ser considerado más como un factor de riesgo que de protección ${ }^{(4,8,16)}$. 
Como se esperaba, el estoicismo fue mayor en los pacientes de más edad siendo posible que el estoicismo aumente debido a la madurez relacionada con la edad que conlleva inevitablemente una mayor aceptación de las pérdidas ${ }^{(17)}$ y una propensión a ocultar el dolor, o a no buscar ayuda para evitar mostrar debilidad ${ }^{(8,17)}$. Del mismo modo, hay autores que lo han explicado como un efecto de cohorte; es decir, que las personas mayores han crecido en una cultura que valoraba la abnegación y la resignación $^{(4,19,20)}$.

Por otro lado, sabemos que las mujeres de nuestra muestra son en promedio 7 años más jóvenes que los hombres, 58 años frente a 65 años, respectivamente, lo que reafirma que los hombres de nuestra muestra sean más estoicos, debido a su mayor edad y al hecho de ser hombres. Con la edad, la repercusión a nivel psicológico es menor, tanto en hombres como en mujeres. De este modo, los hombres ancianos manifiestan menos malestar psicológico, ansiedad y depresión, y las mujeres de mayor edad también presentan menos malestar, somatización y ansiedad, y, además, se vuelven más resignadas (más estoicas).

Sabemos por la literatura que los pacientes deprimidos presentan una mayor amplificación del dolor, menor adherencia a los tratamientos, y cierta indefensión e incapacidad para hacer frente a los problemas ${ }^{(16)}$. Además, algunas personas de mayor edad tienen creencias sesgadas sobre la depresión y la petición de ayuda, fruto de actitudes más culturales que estoicas. La incertidumbre, el temor a la muerte, el dolor y las limitaciones de la enfermedad inducen en los pacientes un sentimiento de preocupación y desesperanza que pueden facilitar la aparición de alteraciones psicopatológicas, tales como los trastornos de ansiedad y la depresión y que modulan su actitud frente la enfermedad ${ }^{(21)}$.

En este sentido, existen numerosos estudios empíricos sobre las estrategias de afrontamiento ante el cáncer que relacionan el estilo de afrontamiento con la progresión de la enfermedad ${ }^{(22)}$. La mayor parte de estos estudios reflejan que los estilos más activos como el espíritu de lucha suelen asociarse a resultados físicos más favorables -especialmente en las fases iniciales de la enfermedad o cuando tiene un buen pronóstico- mientras que estrategias como la resignación o la indefensión se relacionan con peores resultados ${ }^{(23)}$. Este tipo de afrontamiento de tipo evitativo, cercano a la conducta estoica es considerado mal adaptativo por algunos autores ${ }^{(24)}$.

Una de las conductas estoicas es la postergación en la visita al médico después de la aparición de los síntomas. En nuestro estudio, los hombres tardaron, de media, 52 días más en acudir al médico que ellas. Las últimas investigaciones sugieren que las personas estoicas pueden informar de menos de los síntomas físicos ya sea porque no identifiquen tales problemas como enfermedad, por miedo a tener una enfermedad grave, o miedo a parecer menos varoniles ${ }^{(7,17,21)}$. Si esto es así, los hombres y las personas mayores probablemente puedan retrasar la ayuda hasta que los síntomas sean más severos e incapacitantes, o se muestren más reticentes a admitir que están enfermos y necesitan ayuda ${ }^{(5)}$. En cualquier caso, se hace necesario promover la comunicación más fluida y la mayor accesibilidad de los profesionales de la salud que garantice un diagnóstico rápido y un tratamiento eficaz. En este sentido, el estoicismo podría estar actuando en contra del proceso terapéutico en una enfermedad de gran dimensión y con mal pronóstico si se diagnostica en estadios avanzados.

En nuestro estudio, no se ha hallado relación entre el estoicismo y las escalas de dolor, seguramente debido a que los pacientes en el momento de la evaluación 
no presentan mucho dolor porque el tumor se ha operado y aún no han iniciado la quimioterapia y, por tanto, es lógico que no se correlacionen de ninguna manera. Hay autores que sugieren que la actitud estoica es una forma en la que las personas mayores afronta los efectos del dolor crónico ${ }^{(24)}$. En un estudio sobre la percepción del dolor ${ }^{(25)}$ sugieren que el estoicismo es más prevalente en pacientes con dolor de baja intensidad, pero es menor probable ante dolor moderado o severo.

\section{Conclusiones}

El estoicismo es un concepto filosófico de interés en su aplicación en el campo de la psico-oncología, a pesar de que sólo podemos analizar las conductas estoicas observables, desligadas del pensamiento estoico que podría -o no- subyacer en tales actitudes. Saber si los pacientes estoicos soportan mejor la adversidad, depende de si tal actitud estoica fuera fruto de una decisión meditada o bien de una falta de competencia emocional o comunicativa, fruto de un estado anímico deprimido o de una incapacidad de pedir ayuda -como es el caso de algunos hombres mayores ${ }^{(26,27)}$.

En nuestro estudio, las conductas estoicas estuvieron asociadas a hombres, personas de edad avanzada y aquéllos con mayor malestar psicológico y depresión, por lo que nos hace pensar que su presencia pueda considerarse más como un factor de riesgo que de protección. Si la actitud estoica es más fruto del miedo (a la enfermedad, a parecer menos varoniles) estamos ante un factor de riesgo. Ante esta situación es importante contar con la información que nos proporciona la familia y reconocer la importancia del apoyo familiar sobre a estas personas que pueden ser de entrada más vulnerables.

La experiencia e incertidumbre del cáncer, potencialmente mortal, supone una de las situaciones imprevistas más difíciles que han de afrontar las personas y sus familias, dejando señales indelebles, tanto en los pacientes, como en familiares y cuidadores. La forma en que la persona afronta la enfermedad depende de la conciencia que tiene de la misma, de su historia personal, del significado que le dé, de las consecuencias que de ella se deriven y, sobre todo, de los recursos de afrontamiento que pueda poner en marcha ante la situación que le está tocando vivir. En este sentido, el estoicismo aparece como una variable con un valor ambivalente que dependerá de la edad del paciente y del contexto familiar en que se encuentra para ser considerado un peligro o una ventaja. Las conductas estoicas podrían favorecer o dificultar el tratamiento, la comunicación con los profesionales e incluso la toma de decisiones compartida.

En cualquier caso, el análisis del contexto familiar y asistencial del paciente, es condición para asegurar una correcta comunicación médico-paciente y la deseable toma de decisión compartida. Establecer modelos de comunicación efectivos entre profesionales y pacientes, basados en la información objetiva y en la empatía, deben modular y evitar aquellas conductas estoicas que aíslen a los pacientes que las presentan. Asimismo, sería interesante poder efectuar un estudio longitudinal para conocer el grado de implicación de las conductas estoicas en la evolución del paciente. 


\section{Referencias bibliográficas}

1. Wagstaff G, Fowledge AM. Stoicism: Its Relation to Gender, Attitudes Toward Poverty, and reactions to emotive material. J Soc Psychol 1995; 135: 181-4. doi: 10.1080/00224545.1995.9711421

2. Becker LC. Human health and stoic moral norms. J Med Philos 2003;28: 221-38. doi: 10.1076/jmep.28.2.221.14206

3. Addis ME, Mahalik, JR. Men, masculinity, and the contexts of help seeking. Am Psychol 2003; 58: 5-14. doi: 10.1037/0003-066X.58.1.5

4. Murray G, Judd F, Jackson H, Fraser C, Komiti A, Pattison P, et al. Big boys don't cry: An investigation of stoicism and its mental health outcomes. Pers Individ Dif 2008;44:136981. doi:10.1016/j.paid.2007.12.005

5. Calderón C, Ferrando PJ, Urbano Lorenzo-Seva U, Carmona-Bayonas A, Jara C, Ayala de la Peña F, et al. Psychometric properties of Liverpool Stoicism Scale (LSS) in a cohort of patients with resected cancer in adjuvant treatment. Ann Psychol 2017;33: 621-9. doi: 10.6018/analesps.33.3.277061

6. Buckley J, Ó Tuama S. "I send the wife to the doctor"- Men's behaviour as health consumers. Int J Consum Stud 2010;34:587-95. doi: 10.1111/j.1470-6431.2010.00908.x

7. Gaitniece-Putane A. Gender and age differences in in emotional intelligence, stoicism and aggression. Baltic J Psychol 2006; 7:26-42.

8. Moore A, Grime J, Campbell P, Richardson J. Troubling stoicism: Sociocultural influences and applications to health and illness behaviour. Health (London) 2013;17: 159-73. doi: 10.1177/1363459312451179

9. Yong HH. Can attitudes of stoicism and cautiousness explain observed age-related variation in levels of self-rated pain, mood disturbance and functional interference in chronic pain patients? Eur J Pain 2006;10: 399-407. doi. 10.1016/j.ejpain.2005.05.004

10. Cleeland CS, Ryan KM. Pain assessment: global use of the Brief Pain Inventory. Ann Acad Med 1994; 23:129-38. [Acceso 15 de enero de 2018] Disponible en: http://www. ncbi.nlm.nih.gov/pubmed/8080219

11. Kumar SP. Utilization of brief pain inventory as an assessment tool for pain in patients with cancer: a focused review. Indian J Palliat Care 2011;17:108-15. doi: 10.4103/09731075.84531

12. Badia X, Muriel C, Gracia A, Núñez-Olarte JM, Perulero N, Gálvez R et al. Validation of the Spanish version of the Brief Pain Inventory in patients with oncological pain. Med Clin (Barc) 2003; 120: 52-9. [Acceso 15 de enero de 2018]. Disponible en: http://www. ncbi.nlm.nih.gov/pubmed/12570914

13. Derogatis LR. BSI 18, Brief Symptom Inventory 18: Administration, scoring and procedures manual. Minneapolis, MN : NCS Pearson, Inc., (C2001.

14. Recklitis CJ, Blackmon JE, Chang G. Validity of the Brief Symptom Inventory-18 (BSI18) for identifying depression and anxiety in young adult cancer survivors: comparison with a structured clinical diagnostic interview. Psychol Assess 2017; 29: 1189-200. doi: $10.1037 /$ pas0000427

15. International ethical guidelines for biomedical research involving human subjects CIOMS Geneva 2002. [Acceso el 15 de enero de 2018] Disponible en: http://www.cioms. ch/publications/layout_guide2002.pdf

16. Moore A, Grime J, Campbell P, Richardson J. Troubling stoicism: Sociocultural influences and applications to health and illness behaviour. Health (London) 2012;17:159-73. doi: $10.1177 / 1363459312451179$ 
17. Judd F, Komiti A, Jackson H. How does being female assist help-seeking for mental health problems?. Aust N Z J Psychiatry 2008;42:24-9. doi: 10.1080/00048670701732681

18. Wrigley S, Jackson H, Judd F, Komiti A. Role of stigma and attitudes toward helpseeking from a general practitioner for mental health problems in a rural town. Aust $\mathrm{N} Z$ J Psychiatry 2005;39:514-21. doi: 10.1111/j.1440-1614.2005.01612.x

19. Corboy D, McLaren S, Jenkins M, McDonald J. The relationship between geographic remoteness and intentions to use a telephone support service among Australian men following radical prostatectomy. Psychooncology 2014; 23: 1259-66. doi: 10.1002/ pon. 3559

20. Stewart D, Harmon K. Mental health services responding to men and their anger. Int J Ment Health Nurs 2004; 13: 249-54. doi: 10.1111/j.1440-0979.2004.00341.x

21. Witte TK, Gordon KH, Smith, PN. Stoicism and Sensation Seeking: Male vulnerabilities for the acquired capability for suicide. J Res Pers 2012;46:384-92. doi: 10.1016/j. jrp.2012.03.004

23. Bao Y, Li L, Guan Y, Wang W, Liu Y, Wang P et al. Prevalence and associated positive psychological variables of anxiety and depression among patients with central nervous system tumors in China : a cross-sectional study. Psychooncology 2017; 26: 262-9. doi: $10.1002 /$ pon. 4128

24. Sharpley CF, Christie DR. H, Bitsika V, Miller BJ. Trajectories of total depression and depressive symptoms in prostate cancer patients receiving six months of hormone therapy. Psychooncology 2017;26:60-6. doi: 10.1002/pon.4100

25. Yeung NC, Lu Q. Affect as a mediator between self-efficacy and quality of life among Chinese cancer survivors in China. Eur J Cancer Care 2014; 23:149-55. doi: 10.1111/ ecc. 12123

26. Helme RD, Gibson SJ. The epidemiology of pain in elderly people. Clin Geriatr Med 2001;17:417-31.

27. Johansson M, Rydén A, Finizia C. Mental adjustment to cancer and its relation to anxiety, depression, HRQL and survival in patients with laryngeal cancer - a longitudinal study. BMC Cancer 2011;11: 283. doi:10.1186/1471-2407-11-283

28. Oh YS. Communications with health professionals and psychological distress in family caregivers to cancer patients: A model based on stress-coping theory. Appl Nurs Res 2017;33:5-9. doi: 10.1016/j.apnr.2016.09.008 